\title{
SLICES OF MAPS AND LEBESGUE AREA $\left({ }^{1}\right)$
}

\author{
BY \\ WILLIAM P. ZIEMER
}

\begin{abstract}
For a large class of $k$ dimensional surfaces, $S$, it is shown that the Lebesgue area of $S$ can be essentially expressed in terms of an integral of the $k-1$ area of a family, $F$, of $k-1$ dimensional surfaces that cover $S$. The family $F$ is regarded as being composed of the slices of $F$. The definition of the $k-1$ area of a surface restricted to one of its slices is formulated in terms of the theory developed by $\mathrm{H}$. Federer, [F3].
\end{abstract}

1. Introduction. There are many results in geometric measure theory that yield an inequality between the $k$ dimensional measure of a set $R \subset E^{n}$ and the integral of the $k-1$ dimensional measure of the intersection of $R$ with the level surfaces of certain real-valued functions defined on $E^{n}$. There are similar results in the theory of Lebesgue area.

In this paper we will show that the Lebesgue area of a large class of $k$ dimensional surfaces, $S$, can be essentially expressed in terms of an integral of the $k-1$ area of a family, $F$, of $k-1$ dimensional surfaces that cover $S$. An analogous result is obtained for the $k$ dimensional Hausdorff measure of a Hausdorff $k$-rectifiable set. We regard the family $F$ as being composed of the slices of the surface and one of the essential parts of our problem is to determine the appropriate definition of the $k-1$ area of a surface restricted to one of its slices. The definition that we employ is expressed in terms of the theory developed by H. Federer, [F3]. In case $k=2$, there are two other definitions considered and we show that they lead to the same results. One definition was created by L. Cesari $[C]$ and it employs the concepts of the Carathéodory theory of prime ends. The other definition depends upon the notion of the length of a light mapping that was introduced in [F2].

For 2 dimensional surfaces, R. Rishel [RL] and R. Fullerton [FN] obtained results that are in the same spirit as ours by employing Cesari's definition of generalized length. However, Rishel's definition of the slice of a surface is different from ours and Fullerton's premature death precluded him from developing his results beyond polyhedral parametric surfaces and continuously differentiable nonparametric surfaces.

Received by the editors June 23, 1970.

AMS 1970 subject classifications. Primary 2880; Secondary 2850.

Key words and phrases. Lebesgue area, slice of a surface, current valued measure, rectifiable set.

(') This work was supported in part by NSF grant GP 19694.

Copyright (C) 1972, American Mathematical Society 
2. Preliminaries and definitions. Euclidean $n$-space will be denoted by $E^{n}$. Lebesgue measure on $E^{n}$ will be denoted by $L_{n}$ and $H^{k}$ will stand for $k$ dimensional Hausdorff measure. If $A \subset E^{n}$, then the measure $H^{k} \mathrm{~L} A$ is defined by $H^{k} L A(E)$ $=H^{k}(A \cap E)$. A set $R \subset E^{n}$ is called Hausdorff $k$-rectifiable if there is a Lipschitzian function $f$ on $E^{k}$ to $E^{n}$ such that

$$
H^{k}(R \text {-range } f)=0 .
$$

We refer the reader to [F4] for a thorough investigation of the properties possessed by Hausdorff $k$-rectifiable sets. Many of these properties will be employed below and the following will be especially useful. If $R \subset E^{n}$ is a Hausdorff $k$-rectifiable set, $g: R \rightarrow E^{m}$ is Lipschitzian, and $j=\min [k, m]$, then $g$ possesses an $\left(H^{k} L R, k\right)$ approximate $j$ dimensional Jacobian $H^{k} L R$ almost everywhere, [F4, 3.2.19]. Denote this Jacobian by ap $J_{j} g$ and in case $k>m=1$, we will use the notation ap $|\nabla g|$. The following theorem is of particular importance, [F4, 3.2.20, 3.2.22].

2.1. THEOREM. If $R \subset E^{n}$ is Hausdorff $k$-rectifiable, $g: R \rightarrow E^{m}$ is Lipschitzian, and $j=\min [k, m]$, then

$$
\int_{R} \operatorname{ap} J_{j} g d H^{k}=\int_{E^{m}} H^{\alpha}\left[g^{-1}(y) \cap R\right] d H^{j}(y)
$$

where $\alpha=\max [0, k-m]$.

Finally, we recall the definition of Hausdorff $k$ dimensional density

$$
\Theta^{k}\left(H^{k} L R, y\right)=\lim _{r \rightarrow 0} \alpha(k)^{-1} r^{-k} H^{k}[R \cap B(y, r)]
$$

where $B(y, r)$ denotes the open $n$-ball of radius $r$ with center at $y$ and $\alpha(k)$ is the volume of the unit $k$-ball in $E^{k}$. If $R$ is Hausdorff $k$-rectifiable, then

$$
\Theta^{k}\left(H^{k}\llcorner R, y)=1\right.
$$

for $H^{k}$ almost all $y \in R$.

We will now show that the Hausdorff $k$ measure of a rectifiable set can be expressed in terms of the integral of the $k-1$ measure of its slices. The proof of this result will establish the method that is basic in the demonstration of the main theorem, 3.3, that appears below.

2.2. TheOREM. Suppose $R \subset E^{n}$ is a Hausdorff $k$-rectifiable set. Then

$$
H^{k}(R)=\sup \left\{\int_{E^{1}} H^{k-1}\left[u^{-1}(r) \cap R\right] d L_{1}(r)\right\}
$$

where the supremum is taken over all Lipschitz functions $u: E^{n} \rightarrow E^{1}$ that have Lipschitz constant 1.

Proof. If $u$ is such a function, then ap $|\nabla u|$ exists and is dominated by 1 at $H^{k} L R$ almost all points. Therefore, by appealing to Theorem 2.1 it is clear that the above supremum is no more than $H^{k}(R)$. 
In order to establish the opposite inequality, it will suffice to show that for every $\varepsilon>0$, there is a function $u: E^{n} \rightarrow E^{1}$ with Lipschitz constant 1 which satisfies

$$
\int_{R} \operatorname{ap}|\nabla u| d H^{k}>(1-\varepsilon) H^{k}(R)
$$

To this end, first recall that $R$ has an $H^{k}$ approximate tangent $k$ plane, $P(y)$, at $H^{k}$ almost all $y \in R,[\mathrm{~F} 4,3.3 .18]$. Moreover, there exists a $H^{k} L R$ measurable function $\alpha$ defined on $R$ with values in the space of Grassmann simple $k$ vectors of unit norm. In addition, for $H^{k}$ almost all $y \in R, P(y)$ is the $k$ dimensional vectorsubspace of $E^{n}$ associated with $\alpha(y),[\mathrm{F} 4,3.2 .25]$. Consequently, from Lusin's Theorem, the density theorem [F4, 2.9.11], and (1), it follows that at $H^{k}$ almost all $y \in R$ there is a set $A \subset R$ such that

(i) $\Theta^{k}\left(H^{k} \mathrm{~L} A, y\right)=1$ and

(ii) $\alpha$ is continuous at $y$ relative to $A$.

Consider a point $y \in R$ where $P(y)$ exists and let $N(y)$ be the $n-k$ plane passing through $y$ that is perpendicular to $P(y)$. For every real number $\beta>0$, let

$$
C(\beta)=\{x: \operatorname{dist}[x, N(y)]>\beta \operatorname{dist}[x, P(y)]\} .
$$

It follows from the definition of the $H^{k}$ approximate tangent $k$ plane, $P(y)$, that

$$
\lim _{r \rightarrow 0} r^{-k} H^{k}[R \cap B(y, r)-C(\beta)]=0
$$

for every $\beta>0$.

Choose $y \in R$ such that $P(y)$ exists, and where (1) and (3) hold. This will be true at $H^{k}$ almost all $y \in R$. Let $\varepsilon>0$. Choose $\zeta(\varepsilon)=\zeta$ (which will be determined later) and select $\beta$ large enough to ensure that

$$
\left|\pi_{y}[(z-y) /|z-y|]\right|>1-\zeta
$$

whenever $z \in C(\beta)$. Here $\pi_{y}: E^{n} \rightarrow P(y)$ denotes the orthogonal projection. From (4), (3), and the equality

$$
\Theta^{k}\left(H^{k}\llcorner R, y)=\Theta^{k}\left(H^{k}\llcorner A, y)=1\right.\right.
$$

follows the existence of $r^{*}(y, \varepsilon)=r^{*}$ such that, for $0<r<r^{*}$,

$$
\begin{aligned}
& H^{k}[A \cap B(y, r) \cap C(\beta)]>(1-\varepsilon)^{1 / 2} H^{k}[R \cap B(y, r)] \text { and } \\
& |\alpha(x)-\alpha(y)|<\zeta \quad \text { whenever } x \in A \cap B(y, r) .
\end{aligned}
$$

Select $0<r<r^{*}$ and define $u(x)=\operatorname{dist}\left[x, E^{n}-B(y, r)\right]$. Since $|\nabla u(x)|=1$ in $B(y, r)$, (5) implies that

$$
\left|\pi_{y}[\nabla u(x)]\right|>1-\zeta \text { for } x \in B(y, r) \cap C(\beta)
$$


If $\zeta$ is chosen sufficiently small, then from (6) and (7) clearly follows $\left|\pi_{x}[\nabla u(x)]\right|$ $>(1-\varepsilon)^{1 / 2}$ for $x \in A \cap B(y, r) \cap C(\beta)$ where $P(x)$ exists. Therefore,

$$
\int_{R \cap B(y, r)} \operatorname{ap}|\nabla u| d H^{k} \geqq(1-\varepsilon)^{1 / 2} H^{k}[A \cap B(y, r) \cap C(\beta)]
$$

and thus (6) leads to

$$
\int_{R \cap B(y, r)} \operatorname{ap}|\nabla u| d H^{k} \geqq(1-\varepsilon) H^{k}[R \cap B(y, r)]
$$

whenever $0<r<r^{*}$.

For $H^{k}$ almost every $y \in R$, consider the family of all $n$-balls $B(y, r)$ where $0<r<r^{*}(y, \varepsilon)$. Then by a covering theorem due to Besicovitch and A. P. Morse that has been generalized in [F4, 2.8.15], there exist a countable number of $n$-balls, $B_{1}, B_{2}, \ldots$, whose closures are disjoint such that $H^{k} L R\left(E^{n}-\bigcup_{i=1}^{\infty} B_{i}\right)=0$. With each $n$-ball $B_{i}$ is associated the function $u_{i}(x)=$ dist $\left[x, E^{n}-B_{i}\right]$ so that (8) is satisfied. Letting $u(x)=\sum_{i=1}^{\infty} u_{i}(x), x \in E^{n}$, it is clear that $u$ has Lipschitz constant 1 and that

$$
\begin{aligned}
(1-\varepsilon) H^{k}(R) & =(1-\varepsilon) H^{k} L R\left(E^{n}\right)=(1-\varepsilon) \sum_{i=1}^{\infty} H^{k} L R\left(B_{i}\right) \\
& \leqq \sum_{i=1}^{\infty} \int_{R \cap B_{i}} \text { ap }\left|\nabla u_{i}\right| d H^{k}=\int_{R} \operatorname{ap}|\nabla u| d H^{k}
\end{aligned}
$$

According to (2), this concludes the proof.

3. Slices and Lebesgue area. In this section we will establish a result concerning Lebesgue area that is analogous to Theorem 2.2. Throughout this section we will consider a continuous map $f: X \rightarrow E^{n}$ of finite Lebesgue area, where $X$ is a $k$ dimensional smooth manifold, $k \leqq n$. We will also assume that $k=2$ or $H^{k+1}[f(X)]=0$.

Our treatment relies heavily on the work of Federer [F3] and the notation and results of that paper will be employed here without change. Thus, the monotonelight factorization $f=l_{f} \circ m_{f}$ will be considered where $l_{f}$ is defined on the middle space, $M_{f}$. Moreover, there is a unique current-valued measure $\mu$ over $M_{f}$ whose total variation, $\|\mu\|$, is equal to the Lebesgue area of $f$. If $T$ is a current, then $M(T)$ is the mass of $T$ and $F(T)$ denotes the flat norm of $T$. The boundary of $T$ is denoted by $\partial T$. Define a measure $\nu$ over $E^{n}$ by $\nu(A)=\|\mu\|\left(l_{f}^{-1}(A)\right)$.

One of the main results of [F3] states that there is an integer valued function, $K$, such that

$$
\mathscr{L}(f)=\int_{E^{n}} K(y) d H^{k}(y)
$$

where $\mathscr{L}(f)$ is the Lebesgue area of $f$. For $y \in E^{n}$ and $r>0$, let $Z(r)$ be the family of components of $l_{f}^{-1}[B(y, r)]$. For $v$ almost all $y \in E^{n}$, there are a finite number of 
"essential" points, $z$, of $l_{f}^{-1}(y)$ with the property that if $r$ is sufficiently small, then with each $V \in Z(r)$ that contains an essential $z$ is associated an integer $m(V)$ such that

$$
K(y)=\sum_{V \in Z(r)}|m(V)| .
$$

Moreover, there is an oriented $k$ dimensional plane, $P(y)$, passing through $y$ such that, for each such $V \in Z(r)$,

$$
\lim _{r \rightarrow 0} r^{-k} F[\mu(V)-m(V) \cdot P(y) \cap B(y, r)]=0 .
$$

3.1. Definition. Let $u: E^{n} \rightarrow E^{1}$ be Lipschitz and let $Z(r)$ be the set of components of $M_{f} \cap\left\{z: u \circ l_{f}(z)<r\right\}$. Define

$$
\lambda(f ; u, r)=\sum_{V \in Z(r)} M[\partial \mu(V)] .
$$

As a function of $r, \lambda(f ; u, r)$ is $L_{1}$ measurable; indeed,

$$
\liminf _{t \rightarrow r^{-}} \lambda(f ; u, t) \geqq \lambda(f ; u, r) .
$$

To see this, choose $r>0$ and let $V_{1}, V_{2}, \ldots$ be the components that constitute $Z(r)$. For $t>r$ let $W_{i}(t)$ be the union of those components of $Z(t)$ that are contained in $V_{i}, i=1,2, \ldots$ The sets $W_{i}(t)$ are nested and increase with $t$. Therefore, since $\bigcup_{t<r} W_{i}(t)=V_{i}$, it follows that, as currents, $\mu\left[W_{i}(t)\right] \rightarrow \mu\left(V_{i}\right)$ weakly as $t \rightarrow r^{-}$. From the facts that $\partial$ is continuous and mass is lower semicontinuous with respect to weak convergence follows

$$
\liminf _{t \rightarrow r^{-}} M\left[\partial \mu\left(W_{i}(t)\right)\right] \geqq M\left[\partial \mu\left(V_{i}\right)\right], \quad i=1,2, \ldots
$$

Hence,

$$
\begin{aligned}
\liminf _{t \rightarrow r^{-}} \lambda(f ; u, t) & \geqq \liminf _{t \rightarrow r^{-}} \sum_{i=1}^{\infty} M\left[\partial \mu\left(W_{i}(t)\right)\right] \\
& \geqq \sum_{i=1}^{\infty} M\left[\partial \mu\left(V_{i}\right)\right]=\lambda(f ; u, r) .
\end{aligned}
$$

3.2. LEMMA. If $f: X \rightarrow E^{n}$ and $u: E^{n} \rightarrow E^{1}$ has Lipschitz constant $N$, then

$$
\int_{-\infty}^{\infty} \lambda(f ; u, r) d L_{1}(r) \leqq N \mathscr{L}(f)
$$

Proof. Let

$$
\gamma(r)=\|\mu\|\left(\left\{z: u \circ l_{f}(z)<r\right\}\right) .
$$

Observe, for $L_{1}$ almost all $r$, that $\gamma^{\prime}(r)<\infty$ and for each $V \in Z(r)$ that $\mu(V)$ is an integral current, [F3, 3.4]. Now by applying [FF, 3.9] to $T=\mu(V)$, the proof proceeds as in $[F 3,3.2]$ and we obtain

$$
M[\partial \mu(V)] \leqq N \liminf _{h \rightarrow 0^{+}} h^{-1}\|\mu(V)\|(\{y: r-h \leqq u(y)<r\})
$$


From this follows

$$
\begin{aligned}
\lambda(f ; u, r) & \leqq N \liminf _{h \rightarrow 0^{+}} h^{-1} \sum_{V \in Z(r)}\|\mu(V)\|(\{y: r-h \leqq u(y)<r\}) \\
& \leqq N \liminf _{h \rightarrow 0^{+}} h^{-1}\|\mu(V)\|\left(\left\{z: r-h \leqq u \circ l_{f}(z)<r\right\}\right) \leqq N \gamma^{\prime}(r) .
\end{aligned}
$$

Our purpose now is to show that the supremum of the left side of 3.2 over all functions $u$ with $N=1$ equals the Lebesgue area of $f$.

To this end let $R$ be the set of those $y \in E^{n}$ for which (10) and (11) hold and for which $0<K(y)<\infty$. Then $R$ is a Hausdorff $k$-rectifiable set and, without loss of generality, we may assume that

$$
\Theta^{k}\left(H^{k} L R, y\right)=1, \quad y \in R .
$$

Choose $y \in R$ and to simplify notation, take $y=0$. Assume $r$ to be taken small enough so that (10) holds. Select an essential $z \in l_{f}^{-1}(0)$ and consider those $V \in Z(r)$ that contain $z$. Let $T_{r}=\mu(V)$ and $P=P(0)$. Let $\pi: E^{n} \rightarrow P$ be the orthogonal projection and define $h_{r}: P \rightarrow P$ by $h_{r}(x)=r^{-1} \cdot x$. The flat norm of a current is not increased under a projection and, for $k$ dimensional currents in $E^{k}$, the flat norm and the mass norm agree. Therefore, it follows from (11) that

$$
\lim _{r \rightarrow 0} r^{-k} M\left[\pi_{\#}\left(T_{r}\right)-m(V) \cdot B(0, r) \cap P\right]=0 .
$$

This implies

$$
\lim _{r \rightarrow 0} M\left[\left(h_{r} \circ \pi\right)_{\#}\left(T_{r}\right)-m(V) \cdot B(0,1) \cap P\right]=0 .
$$

Again, from the continuity of $\partial$ and the lower semicontinuity of mass, for $\eta>0$ and all sufficiently small $r$,

$$
M\left[\left(h_{r} \circ \pi\right)_{\#}\left(\partial T_{r}\right)\right] \geqq|m(V)| M[\partial(B(0,1) \cap P)]-\eta .
$$

Thus, for all small $r$,

$$
\begin{aligned}
M\left(\partial T_{r}\right) & \geqq M\left[\partial \pi_{\#}\left(T_{r}\right)\right]=M\left[\left(h_{r} \circ \pi\right)_{\#}\left(\partial T_{r}\right)\right] r^{k-1} \\
& \geqq\left[|m(V)|-\eta \alpha(k-1)^{-1}\right] M[\partial(B(0, r) \cap P)] .
\end{aligned}
$$

Choose $\delta>0$. It follows from (10) and (13) that, for $y \in R$, there is $r^{*}=r^{*}(y, \delta)$ such that if $B=B(y, r), 0<r<r^{*}$, and $u(x)=-\operatorname{dist}\left(x, E^{n}-B\right)$, then

$$
\lambda(f ; u,-t)=\sum_{V \in Z(-t)} M[\partial \mu(V)] \geqq[K(y)-\delta] M[\partial(B(y, t) \cap P)]
$$

where $Z(-t)$ is the set of components of $\left\{z: u \circ l_{f}(z)<-t\right\}$ and $0<t<r$. Therefore,

$$
\int_{-\infty}^{\infty} \lambda(f ; u, t) d L_{1}(t) \geqq[K(y)-\delta] \alpha(k) r^{k} .
$$

Define a measure $\zeta$ over $E^{n}$ by

$$
\zeta(E)=\int_{E \cap R} K(y) d H^{k}(y)
$$


for every Borel set $E$. Then

$$
\zeta\left(E^{n}\right)=\mathscr{L}(f)
$$

indeed, $\zeta=\nu$, but we will not use this fact. Appealing to [F4, 2.9.8] we have for $H^{k}$ almost all $y \in R$, that

$$
\lim _{r \rightarrow 0} \zeta[B(y, r)] / H^{k} L R[B(y, r)]=K(y) .
$$

For $\varepsilon>0$ and $y \in R$, it follows from (12), (14), and (16) that there exists $r^{*}(y, \varepsilon)=r^{*}$ such that

$$
\int_{-\infty}^{\infty} \lambda(f ; u, t) d L_{1}(t) \geqq(1-\varepsilon) \zeta[B(y, r)]
$$

whenever $0<r<r^{*}$. Consider the family of $n$-balls, $B(y, r), y \in R$ and $0<r<r^{*}(y, \varepsilon)$. Appealing to the covering theorem [F4, 2.8.15], there exist balls $B_{1}, B_{2}, \ldots$ whose closures are disjoint and that have the property

$$
\zeta\left(E^{n}-\bigcup_{i=1}^{\infty} B_{i}\right)=0 .
$$

As in the proof of 2.2 we define

$$
u(x)=\sum_{i=1}^{\infty} u_{i}(x), \quad x \in E^{n},
$$

where $u_{i}(x)=-\operatorname{dist}\left(x, E^{n}-B_{i}\right), i=1,2, \ldots$ Then $u$ has Lipschitz constant 1 and $\lambda(f ; u, r)=\sum_{i=1}^{\infty} \lambda\left(f ; u_{i}, r\right), r \in E^{1}$. Therefore, (17) and (18) yield

$$
\begin{aligned}
\int_{-\infty}^{\infty} \lambda(f ; u, r) d L_{1}(r) & =\sum_{i=1}^{\infty} \int_{-\infty}^{\infty} \lambda\left(f ; u_{i}, r\right) d L_{1}(r) \\
= & \sum_{i=1}^{\infty}(1-\varepsilon) \zeta\left(B_{i}\right)=(1-\varepsilon) \zeta\left(E^{n}\right)=(1-\varepsilon) \mathscr{L}(f) .
\end{aligned}
$$

Thus, the following theorem has been established.

3.3. TheOREM. Suppose $f: X \rightarrow E^{n}$ has finite Lebesgue area and suppose $k=2$ or $H^{k+1}[f(X)]=0$. Then $\sup \left\{\int_{-\infty}^{\infty} \lambda(f ; u, r) d L_{1}(r)\right\}=\mathscr{L}(f)$ where the supremum is taken over all functions $u: E^{n} \rightarrow E^{1}$ that have Lipschitz constant 1 .

4. Mappings from a 2-cell. The results of [F3] are also valid in case $X$ is a manifold with boundary, [G], [M], and therefore Theorem 3.3 also holds in this case. In this section we consider the special situation when $X$ is the unit square, $Q$, in $E^{2}$ for then there are two other reasonable definitions for the 1-area or "length" of $f$ restricted to a slice determined by $u$. In order for 3.3 to remain valid, Definition 3.1 of $\lambda(f ; u, r)$ is modified so that only those components $V \in Z(r)$ are considered for which closure $V \cap m_{f}($ bdry $Q)=0$. Let $C(r)$ be the set of such components.

4.1. Definition. If $U$ is a subset of a separable metric space, let

$$
\delta(U)=\text { bdry } U \cap\{z: \text { dimension (bdry } U, z)>0\} \text {. }
$$


4.2. Definition. For $V \in C(r)$, let

$$
|V|=\int_{E^{n}} N\left[l_{f}, \delta(V), y\right] d H^{1}(y)
$$

where $N\left[l_{f}, \delta(V), y\right]$ denotes the number of points (possibly $\infty$ ) in $l_{f}^{-1}(y) \cap \delta(V)$. Define

$$
\rho(f ; u, r)=\sum_{v \in C(r)}|V| .
$$

Similarly, for $V \in C(r)$, let $\|V\|$ denote the length of $f$ restricted to the boundary of $m_{f}^{-1}(V)$ as defined by Cesari in [C, 20.2]. Define

$$
\sigma(f ; u, r)=\sum_{v \in C(r)}\|V\| .
$$

Finally, define

$$
\mathscr{C}(f)=\sup \left\{\int_{-\infty}^{\infty} \sigma(f ; u, r) d L_{1}(r)\right\}
$$

where the supremum is taken over all functions $u: E^{n} \rightarrow E^{1}$ that have Lipschitz constant 1 . By replacing $\sigma(f ; u, r)$ by $\rho(f ; u, r)$, we define $\mathscr{D}(f)$ in a similar manner.

The following result is an immediate consequence of $[C, 20.5]$ and $[R L, 1.6]$.

4.2. THEOREM. If $f: Q \rightarrow E^{n}$ is continuous, then $\mathscr{L}(f) \geqq \mathscr{C}(f) \geqq \mathscr{D}(f)$.

In order to prove that $\mathscr{D}(f) \geqq \mathscr{L}(f)$, we first observe that if $f$ and $g$ are Fréchet equivalent maps of $Q$ into $E^{n}$, then $\mathscr{D}(f)=\mathscr{D}(g)$. The same is true for the functionals $\mathscr{L}$ and $\mathscr{C},[\mathrm{C}, 31.7]$. These facts will be very useful since we later employ Morrey's representation theorem. Undoubtedly, it is possible to prove $\mathscr{D}(f) \geqq \mathscr{L}(f)$ without resorting to Morrey's theorem, but we prefer this method since we anticipate an application of (24) below in future work.

In this regard recall that a continuous map $f: Q \rightarrow E^{n}$ is almost conformal provided that

(i) the coordinate functions of $f$ are absolutely continuous in the sense of Tonelli (ACT) on $Q$, and their partial derivatives are square integrable on $Q$, and

(ii) the partial derivatives $D_{1} f, D_{2} f$ satisfy $D_{1} f(x) \cdot D_{2} f(x)=0$ for $L_{2}$ almost all $x \in Q$.

The formal differential, $d f$, which is defined as the linear transformation associated with the matrix of partial derivatives of $f$, exists for $L_{2}$ almost all points in $Q$. Thus, for all such $x, d f(x)$ is a linear transformation from $E^{2}$ into $E^{n}$ and it induces a linear transformation on the space of Grassmann 2-vectors. Thus, if $\alpha$ is a 2-vector in $E^{2}$, then $d f(x)(\alpha)=d f(x, \alpha)$ is a 2-vector in $E^{n}$. Later, we will also use the fact that if $\left\{f_{i}\right\}$ is a sequence of mollifiers of $f$, then

(i) $f_{i} \rightarrow f$ uniformly on compact subsets of $Q$ and

(ii) $\int_{Q}\left|d f_{i}-d f\right|^{2} d L_{2} \rightarrow 0$ where $|d f(x)|$ denotes the norm of $d f(x)$. 
If $u: E^{n} \rightarrow E^{1}$ is Lipschitz and $f: Q \rightarrow E^{n}$ is almost conformal, then it is not difficult to show that $F=u \circ f$ is ACT on $Q$ and $|\nabla F|$ is square integrable on $Q$. Moreover, the following facts were established in $[\mathrm{Z}]$ :

(i) If $g$ is square integrable, then

$$
\int_{Q}|\nabla F| g d L_{2}=\int_{E^{1}} \int_{F^{-1}(r)} g(y) d H^{1}(y) d L_{1}(r) .
$$

(ii) If $W$ is a component of $\{x: F(x)<r\}$, then for $L_{1}$ almost all $r$, $W$ has finite perimeter. That is, if $W$ is considered as a current, then in fact, it is an integral current.

(iii) Let $\beta(W)$ be the reduced boundary of $W$, that is, $\beta(W)$ consists of those points $x$ where $n(x)$, the exterior normal to $W$, exists at $x$. Observe that $\beta(W) \subset$ bdry $W \subset F^{-1}(r)$. Following the proof of $[Z, 3.3]$, we have $H^{1}[\beta(W)-\delta(W)]=0$.

4.3. LemMA. If $f: Q \rightarrow E^{n}$ is almost conformal and $u: E^{n} \rightarrow E^{1}$ is Lipschitz, then for $L_{1}$ almost all $r, f^{-1}(y)$ is totally disconnected for $H^{1}$ almost all $y \in u^{-1}(r)$.

Proof. In view of the fact that the coordinate functions of $f$ are ACT on $Q$, it follows that there is a countable set of vertical line segments in $Q, \Lambda_{1}$, such that $\Lambda_{1}$ is dense in $Q$ and if $\lambda \in \Lambda_{1}$ then $H^{1}[f(\lambda)]<\infty$. Likewise, there is a set $\Lambda_{2}$ corresponding to the horizontal direction. Setting $\Lambda=\Lambda_{1} \cup \Lambda_{2}$, it follows that if $N=f(\Lambda)$ then $H^{2}[N]=0$. However, $[\mathrm{F} 1,3.2]$ or 2.1 implies that $H^{1}\left[N \cap u^{-1}(r)\right]$ $=0$ for $L_{1}$ almost all $r$. Hence, if $y \notin N \cap u^{-1}(r)$, then $f^{-1}(y)$ has only point components.

4.4. LEMMA. With the same hypotheses as $4.3, \lambda(f ; u, r) \leqq \rho(f ; u, r)$ for $L_{1}$ almost all $r \in E^{1}$.

Proof. Let $\alpha$ be a continuous function on $Q$ whose values are unit Grassmann 2-vectors in $E^{2}$. Select $r \in E^{1}$ so that the results of (20) and 4.3 hold, and let $W$ be a component of $\{x: F(x)<r\}$ such that (closure $W$ ) $\cap$ bdry $Q=0$. Since the mollifiers, $f_{i}$, of $f$ are $C^{\infty},(19)$ implies for every $C^{\infty}$ differential 2-form $\varphi$ that

$$
\begin{aligned}
\lim _{i \rightarrow \infty} f_{i \#}(W)(\varphi) & =\lim _{i \rightarrow \infty} \int_{W} \varphi\left[f_{i}(x)\right] \cdot d f_{i}(x, \alpha(x)) d L_{2}(x) \\
& =\int_{W} \varphi[f(x)] \cdot d f(x, \alpha(x)) d L_{2}(x)
\end{aligned}
$$

On the other hand, $[\mathrm{F} 3,3.4]$ implies that, for $L_{1}$ almost all $r, \lim _{i \rightarrow \infty} f_{i_{\sharp 1}}(W)(\varphi)$ $=\mu(V)(\varphi)$ where $V=m_{f}(W)$. Thus,

$$
\mu(V)(\varphi)=\int_{W} \varphi[f(x)] \cdot d f(x, \alpha(x)) d L_{2}(x) .
$$


Since $\int_{Q}\left|d f_{i}-d f\right|^{2} d L_{2} \rightarrow 0$ it follows from [(20)(i)] that there exists a subsequence (which will still be denoted as $\left\{d f_{i}\right\}$ ) such that

$$
\int_{F-1(r)}\left|d f_{i}-d f\right| d H^{1} \rightarrow 0
$$

From Hölder's inequality and (i) of (20), it is clear that (23) holds for $L_{1}$ almost all $r$. Since $\beta(W) \subset F^{-1}(r)$ for all $r$ under consideration, (19), (iii) of (20), (21), (22), (23), and the Gauss-Green Theorem [F4, 4.5.6] imply that, for $L_{1}$ almost all $r$,

$$
\begin{aligned}
\partial \mu(V)(\varphi) & =\lim _{i \rightarrow \infty} \partial f_{i_{\#}}(W)(\varphi)=\lim _{i \rightarrow \infty} f_{i_{\sharp}}(\partial W)(\varphi) \\
& =\lim _{i \rightarrow \infty} \int_{\beta(W)} \varphi\left[f_{i}(x)\right] \cdot d f_{i}(x, v(x)) d H^{1} \\
& =\int_{B(W)} \varphi[f(x)] \cdot d f(x, v(x)) d H^{1}(x) \\
& =\int_{B(W) \cap \delta(W)} \varphi[f(x)] \cdot d f(x, v(x)) d H^{1}(x)
\end{aligned}
$$

where $v(x)$ is the unit vector perpendicular to $n(x)$ chosen so that $v(x) \wedge n(x)=\alpha(x)$. Since the partial derivatives of the coordinate functions of $f$ exist $L_{2}$ almost everywhere in $Q$, we apply [F4, 3.1.8] to find disjoint sets $Q=\bigcup_{i=0}^{\infty} A_{i}$ such that $L_{2}\left(A_{0}\right)$ $=0$ and $f$ restricted to $A_{i}, i>0$, is Lipschitzian. From [FU, Theorem 3(d)] and (i) of (20), we may assume that $H^{1}\left[\delta(W) \cap A_{0}\right]=0$. Thus, [F4, 3.2.20] and (24) yield

$$
M[\partial \mu(V)] \leqq \int_{E^{n}} N[f, \delta(W), y] d H^{1}(y) .
$$

However, 4.3 allows us to assume that

$$
\int_{E^{n}} N[f, \delta(W), y] d H^{1}(y)=\int_{E^{n}} N\left[l_{f}, \delta(V), y\right] d H^{\prime}(y)
$$

and the lemma follows directly from this.

4.5. TheOrem. Suppose $f: Q \rightarrow E^{n}$ has finite Lebesgue area and, in addition, suppose $f$ has the property that for each $y \in E^{n}$, no component of $f^{-1}(y)$ disconnects $Q$. Then $\mathscr{C}(f)=\mathscr{D}(f)=\mathscr{L}(f)$.

Proof. With the conditions imposed on $f$ it follows that the middle space, $M_{f}$, is either a 2-cell or a 2-sphere, [R, II.2.91].

In the event that $M_{f}$ is a 2-cell, Morrey's representation theorem [MO1], [MO2] asserts the existence of an almost conformal map $g: Q \rightarrow E^{n}$ that is Fréchet equivalent to $f$. Now $\mathscr{D}(f)=\mathscr{D}(g)$ and $\mathscr{L}(f)=\mathscr{L}(g)$ while $4.2,4.4$, and 3.3 imply that $\mathscr{D}(g)=\mathscr{L}(g)$.

If $M_{f}$ is a 2 -sphere, then $f$ has a Fréchet equivalent $g: Q \rightarrow E^{n}$ with the property that $g$ is constant on the boundary of $Q$ while $f$ is not constant on any nondegenerate continuum in the interior of $Q$, [R, II.3.28]. However, this case is 
treated essentially the same way as the preceding one and, thus, the proof is complete.

In order to establish Theorem 4.5 for any map $f: Q \rightarrow E^{n}$ of finite Lebesgue area, it will suffice to prove that $\mathscr{D}$ is cyclically additive because it is known that Lebesgue area possesses this property, [R, V.2.55].

Let $C_{1}, C_{2}, \ldots$ be the cyclic elements of $M_{f}$ and let $r_{i}: M_{f} \rightarrow C_{i}$ be the monotone retraction, [R, II.2.40]. The mappings $f_{i}=l_{f} \circ r_{i} \circ m_{f}$ are the cyclic components of $f$ and they satisfy the hypotheses of Theorem 4.5. Let $C_{1}, C_{2}, \ldots, C_{k}$ be any finite number of cyclic elements of $M_{f}$. It will be sufficient to show that

$$
\mathscr{D}(f) \geqq \sum_{i=1}^{k} \mathscr{D}\left(f_{i}\right)
$$

in view of the fact that $\mathscr{D}(f) \leqq \sum_{i=1}^{\infty} \mathscr{D}\left(f_{i}\right)$, which follows from the cyclic additivity of $\mathscr{L}, 4.2$, and 4.5 .

Choose $\varepsilon>0$. From the construction that appears in the proof of Theorem 3.3, it follows that there is a function $u: E^{n} \rightarrow E^{1}$ with Lipschitz constant 1 such that, for $i=1,2, \ldots, k$,

$$
\int_{-\infty}^{\infty} \lambda\left(f_{i} ; u, r\right) d L_{1}(r)>\mathscr{L}\left(f_{i}\right)-\varepsilon / k
$$

In order to see this we will only consider the case of $k=2$, since the general case is handled in the same way and is no more difficult except for complications in notation. Let $\zeta_{i}$ be the measure associated with $f_{i}$ as in (15), $i=1,2$, and let $R_{i}$ be the Hausdorff 2-rectifiable set that is determined by $f_{i}$ as in the proof of Theorem 3.3. Choose compact sets $K_{1} \subset R_{1}-R_{2}, K_{2} \subset R_{2}-R_{1}$, and $K_{3} \subset R_{1} \cap R_{2}$ such that

$$
\begin{gathered}
\zeta_{1}\left(K_{1}\right)>\zeta_{1}\left(R_{1}-R_{2}\right)-\varepsilon / 3, \quad \zeta_{2}\left(K_{2}\right)>\zeta_{2}\left(R_{2}-R_{1}\right)-\varepsilon / 3, \\
\zeta_{i}\left(K_{3}\right)>\zeta_{i}\left(R_{1} \cap R_{2}\right)-\varepsilon / 3, \quad i=1,2 .
\end{gathered}
$$

Let $U_{i}$ be open sets that are mutually disjoint that contain $K_{i}, i=1,2,3$. For each $y \in K_{i}$ and all sufficiently small balls centered at $y$, (17) holds with $f$ and $\zeta$ replaced by $f_{i}$ and $\zeta_{i}, i=1,2$. Similarly, at $H^{2}$ almost all $y \in K_{3}$, (17) is satisfied simultaneously for $i=1,2$ for all sufficiently small balls centered at $y$. We may assume that all balls considered are contained in some $U_{i}, i=1,2,3$. Thus, we have a Vitali cover of $K=\bigcup_{i=1}^{3} K_{i}$ and, therefore, there exist balls $B_{1}, B_{2}, \ldots$ such that

$$
H^{2} L K\left[E^{n}-\bigcup_{i=1}^{\infty} B_{i}\right]=0 .
$$

As in the proof of Theorem 3.3, we define a Lipschitz function $u: E^{n} \rightarrow E^{1}$ in terms of the $B_{i}$. Since $\zeta_{i}$ is absolutely continuous with respect to $H^{2}$, it follows for $i=1,2$ that

$$
\begin{aligned}
\int_{-\infty}^{\infty} \lambda\left(f_{i} ; u, r\right) d L_{1}(r) & \geqq \sum_{j=1}^{\infty}(1-\varepsilon) \zeta_{i}\left(B_{j}\right)=(1-\varepsilon) \zeta_{i}\left(K_{i} \cup K_{3}\right) \\
& \geqq(1-\varepsilon)\left[\zeta_{i}\left(R_{i}\right)-2 \varepsilon / 3\right]=(1-\varepsilon)\left[\mathscr{L}\left(f_{i}\right)-2 \varepsilon / 3\right] .
\end{aligned}
$$


By redefining $\varepsilon$ appropriately, (26) is now established. Therefore, (26), 4.4, and 4.5 imply that

$$
\int_{-\infty}^{\infty} \rho\left(f_{i} ; u, r\right) d L_{1}(r)>\mathscr{D}\left(f_{i}\right)-\varepsilon / k, \quad i=1,2, \ldots, k .
$$

Let $W$ be a component of $\{x: u \circ f(x)<r\}$ and let $V=m_{f}(W)$. Now $r_{i}(V)=V \cap C_{i}$ and the boundary of $V \cap C_{i}$ relative to $C_{i}$ is contained in (bdry $V$ ) $\cap C_{i}$. Consequently, if $V_{i}=V \cap C_{i}, \delta\left(V_{i}\right) \subset \delta(V), i=1,2, \ldots, k$. Any two cyclic elements intersect at most in one point, [R, II.2.24], and therefore

$$
N\left[l_{f}, \delta(V), y\right] \geqq \sum_{i=1}^{k} N\left[l_{f}, \delta\left(V_{i}\right), y\right]
$$

for all but finitely many $y \in E^{n}$. Hence,

$$
\rho(f ; u, r) \geqq \sum_{i=1}^{k} \rho\left(f_{i} ; u, r\right)
$$

and (27) now leads to

$$
\begin{aligned}
\mathscr{D}(f) & \geqq \int_{-\infty}^{\infty} \rho(f ; u, r) d L_{1}(r) \\
& \geqq \sum_{i=1}^{k} \int_{-\infty}^{\infty} \rho\left(f_{i} ; u, r\right) d L_{1}(r)>\sum_{i=1}^{k} \mathscr{D}\left(f_{i}\right)-\varepsilon .
\end{aligned}
$$

Since $\varepsilon$ was arbitrarily chosen, (25) is now established and we have established that if $f: Q \rightarrow E^{n}$ has finite Lebesgue area, then

$$
\mathscr{L}(f)=\mathscr{C}(f)=\mathscr{D}(f) .
$$

Moreover, by considering $u: E^{n} \rightarrow E^{1}$ as the distance function from a fixed hyperplane in $E^{n}$, it follows from Theorems 4.6 and 7.16 of [F2] that there is a constant $k$ such that $\mathscr{L}(f) \leqq k \mathscr{D}(f)$. Thus, if $\mathscr{D}(f)<\infty$ then so is $\left.\mathscr{L}(f)<\infty{ }^{2}\right)$. Therefore 4.2 and (28) yield the following result.

4.6. THEOREM. If $f: Q \rightarrow E^{n}$ is continuous, then $\mathscr{L}(f)=\mathscr{C}(f)=\mathscr{D}(f)$.

The results and techniques employed by Slepian [S1], [S2] will allow the extension of this theorem to maps defined on any 2-dimensional manifold.

\section{BIBLIOGRAPHY}

[C] L. Cesari, Surface area, Ann. of Math. Studies, no. 35, Princeton Univ. Press, Princeton, N. J., 1956. MR 17, 596.

[F1] H. Federer, Some integral geometric theorems, Trans. Amer. Math. Soc. 77 (1954), 238-261. MR 16, 163.

[F2] —-, On Lebesgue area, Ann. of Math. (2) 61 (1955), 289-353. MR 16, 683.

[F3] — Currents and area, Trans. Amer. Math. Soc. 98 (1961), 204-233. MR 23 \#A1006.

$\left.{ }^{2}\right)$ The author is indebted to the referee for this observation. 
[F4] H Federer, Geometric measure theory, Die Grundlehren der math. Wissenschaften, Band 153, Springer-Verlag, New York, 1969. MR 41 \#1976.

[FF] H. Federer and W. H. Fleming, Normal and integral currents, Ann. of Math. (2) 72 (1960), 458-520. MR 23 \#A588.

[FU] B. Fuglede, Extremal length and functional completion, Acta Math. 98 (1957), 171-219. MR 20 \#4187.

[FN] R. E. Fullerton, The Cesari-Cavalieri area of a surface, Duke Math. J. 30 (1963), 151-160. MR 26 \#2581.

[G] R. F. Gariepy, Current valued measures and Geöcze area, Ph.D. Thesis, Wayne State University, Detroit, Mich., 1969; see also Trans. Amer. Math. Soc. (to appear).

[M] J. H. Michael, The convergence of measures on parametric surfaces, Trans. Amer. Math. Soc. 107 (1963), 140-152. MR 26 \#3874.

[MO1] C. B. Morrey, A class of representations of manifolds. I, II, Amer. J. Math. 55 (1933), 683-707; ibid. 56 (1934), 275-293.

[MO2] — An analytic characterization of surfaces of finite Lebesgue area. I, II, Amer. J. Math. 57 (1935), 692-702; ibid. 58 (1936), 313-322.

[R] T. Radó, Length and area, Amer. Math. Soc. Colloq. Publ., vol. 30, Amer. Math. Soc., Providence, R. I., 1948. MR 9, 505.

[RL] R. Rishel, Area as the integral of lengths of contours, Trans. Amer. Math. Soc. 97 (1960), 95-119. MR 22 \#9574.

[S1] P. Slepian, Theory of Lebesgue area of continuous maps of 2-manifolds into n-space, Ann. of Math. (2) 68 (1958), 669-689. MR 20 \#5271.

[S2] —, On the Lebesgue area of a doubled map, Pacific J. Math. 8 (1958), 613-620. MR 24 \#A2003.

[Z] W. Ziemer, Some lower bounds for Lebesgue area, Pacific J. Math. 19 (1966), 381-390. MR 34 \#2830.

Department of Mathematics, Indiana University, Bloomington, Indiana 47401

Department oe Mathematics, University of Michigan, Ann Arbor, Michigan 48104 